\title{
Signals from SABR-COMET time to move on to phase III studies
}

\author{
Michael T. Milano ${ }^{1}$, Amit K. Chowdhry ${ }^{1}$, Joseph K. Salama ${ }^{2}$, Steven J. Chmura ${ }^{3}$ \\ ${ }^{1}$ Department of Radiation Oncology, University of Rochester Medical Center, Rochester, NY, USA; ${ }^{2}$ Department of Radiation Oncology, Duke \\ University, Durham, NC, USA; ${ }^{3}$ Department of Radiation and Cellular Oncology, The University of Chicago, Chicago, IL, USA \\ Correspondence to: Michael T. Milano, MD, PhD. Department of Radiation Oncology, University of Rochester Medical Center, 601 Elmwood Avenue, \\ Box 647, Rochester, NY 14642, USA. Email: michael_milano@urmc.rochester.edu. \\ Provenance: This is an invited article commissioned by the Section Editor Dr. Feng Zhao (Department of Radiation Oncology, The First Affiliated \\ Hospital, College of Medicine, Zhejiang University, Hangzhou, China). \\ Comment on: Palma DA, Olson R, Harrow S, et al. Stereotactic ablative radiotherapy versus standard of care palliative treatment in patients with \\ oligometastatic cancers (SABR-COMET): a randomised, phase 2, open-label trial. Lancet 2019;393:2051-8.
}

Submitted Sep 20, 2019. Accepted for publication Sep 29, 2019.

doi: 10.21037/atm.2019.09.152

View this article at: http://dx.doi.org/10.21037/atm.2019.09.152

Oligometastases represent the clinical state where metastases are limited in number and extent, and potentially amenable to definitive metastases-directed (i.e., surgical or ablative) treatment (1). Systemic therapy is the standard treatment for metastatic cancer, though over the past decade, emerging data have supported the role of surgery or radical irradiation for oligometastatic patients $(2,3)$. The notion that metastasis-directed therapy could improve cancer control and survival outcomes date back decades (4-6), with Hellman and Weichselbaum initially coining the term "oligometastases" and formulating and refining the hypothesis in 1995 (7).

The recently published, multi-national (ten institutions in Canada, Netherlands, UK, and Australia) SABRCOMET randomized phase II screening trial is the first published randomized study that enrolled patients with oligometastases from any primary site, metastasized to any organ/tissue (8). Patients with a controlled primary tumor and 1-5 metastases (at least 1 extracranial and excluding other than femoral metastases) with no more than 3 metastases in any one organ were eligible. Patients were to receive standard of care therapy, and be randomized 2:1 to receive metastasis-directed stereotactic ablative body radiotherapy [SABR; synonymous with stereotactic body radiotherapy (SBRT)] or not ( $\mathrm{n}=66$ patients in the treatment arm and $n=33$ patients in the control arm). Choice of systemic therapy agents (if any) were left to the discretion of the treating physicians, with approximately $50 \%$ receiving systemic therapy. The patients in the SABR arm experienced a significantly increased median progressionfree survival (PFS; 12 vs. 6 months, $\mathrm{P}=0.001$ ). Furthermore, the median overall survival was longer in the treatment arm: 41 vs. 28 months, $\mathrm{P}=0.09$.

A $\mathrm{P}$ value of 0.09 met the study's endpoint of $\mathrm{P}<0.20$ for the randomized phase 2 screening design (9). In such a design, the alpha and beta are often higher than traditional trials in order to ascertain whether further confirmatory trials are warranted. The $\mathrm{P}$ value was chosen to be 2 -sided to allow for the possibility of inferior outcomes associated with SABR. Had the $\mathrm{P}$ value resulted in a value $>0.20$, that finding would suggest that a phase III trial was not warranted due to lack of significant difference between the study arms. Notably, the American Statistical Association emphasizes the importance of estimation, to be used in conjunction with $\mathrm{P}$ values (10). A $\mathrm{P}<0.2$, coupled with a clinically meaningful hazard ratio (i.e., such as the SABR-COMET hazard ratio for death of 0.57 with $95 \%$ confidence interval $0.30-1.10$ ) is compelling. These findings are interesting and suggest an improvement in overall survival with SBRT for oligometastases may be seen if confirmatory clinical trials are conducted with rigorous design and stratification. As planned, the authors have opened two Phase III studies_one specific to patients with 1-3 oligometastases and another specific to patients with 4-10 oligometastases (11). In addition, the study team opted to amend the SABR-COMET protocol to allow for reevaluation after 10-year of follow-up.

One of many limitations of the SABR-COMET 
trial, discussed by the study authors, is the accrual of a heterogeneous patient population. The primary site, histology, sites of metastases, number of lines of prior systemic therapy, and current (or lack of) use of systemic therapy after SABR were not stratified factors in this phase 2 trial design. Such heterogeneity is beneficial in allowing greater patient accrual over a shorter duration of time, but prohibits conclusions of the treatments effectiveness (i.e., metastasis directed SABR may benefit one histologic subgroup over another). Notably prospective Phase I, single-arm pilot, and single-arm Phase II studies from the University of Rochester (12,13), University of Chicago (14), Mt Sinai Hospital (15), University of Pittsburgh (16) and other institutions utilized a similar strategy of relatively broad inclusion criteria to maximize accrual.

The median follow-up of the SABR-COMET trial was just over 2-year with some patient outcomes reported past the 5-year mark. For patients with oligometastatic disease, follow-up beyond 2 years may provide interesting insight. For example, the University of Rochester recently published 10-year outcomes of 48 patients treated with hypofractionated stereotactic radiotherapy for oligometastases from breast cancer (17), showing promising long-term survival even among patients with extra-osseous (i.e., parenchymal) metastases. In subgroup analysis, patients with bone-only disease fared better than those with visceral metastases-with 5- and 10-year overall survival rates of $83 \%$ and $75 \%$ vs. $31 \%$ and $17 \%$, respectively $(\mathrm{P}=0.002)$. In a separate analysis of 82 patients with oligometastases from primary sites other than breast or prostate cancer (18), the 5 - and 10 -year overall survival rates were $13.4 \%$, and $7.3 \%$, respectively. The survival curve for non-breast/non-prostate cancer oligometastases showed that long-term survival was possible after SBRT, and appeared to plateau around the 5-year mark, suggesting potential for cure in a select subset of patients. Interestingly, a greater tumor burden (as measured by net tumor volume) was a significantly adverse factor for OS.

Another limitation of the SABR-COMET is that while patients with 1-5 oligometastases were eligible, only $17 \%$ of patients had 4-5 metastases. As such, the results of SABR-COMET may be most relevant to patients with 1-3 oligometastases; this prompted the investigators to design separate trials of SBRT for 1-3 and 4-10 oligometastases (mentioned above) for the subsequent SABR-COMET studies. It is also notable that $41 \%$ of patients had breast $(20 \%)$ or prostate $(21 \%)$ cancer in the treatment arm compared to $21 \%$ of patients with breast $(15 \%)$ or prostate
(6\%) cancer in the control arm. Using chi square tests, this distribution is different for prostate cancer $(\mathrm{P}=0.054)$ and for breast or prostate cancer $(\mathrm{P}=0.052)$, emphasizing the need for subsequent phase 3 trials to stratify by histology, as patients with oligometastases from breast or prostate cancer experience better outcomes $(13,16,19-21)$. In the forthcoming SABR-COMET studies, the investigators will stratify patients based upon histology: prostate, breast, or renal cancers $v s$. all other histologies (11).

A few randomized phase II studies of metastasis-directed radiotherapy for specific cancers/histologies have recently been published. A randomized phase II study of systemic therapy with $v s$. without radiofrequency ablation (RFA) for non-resectable colorectal liver metastases [European Organisation for Research and Treatment of Cancer (EORTC) 40004 study] showed that metastasis-directed RFA significantly prolonged progression-free survival (hazard ratio $=0.63,95 \%$ confidence interval $0.42-0.95$, $\mathrm{P}=0.025)$; median overall survival was similar between arms, though the trial was not designed to detect survival differences, and the survival in the control arm (no RFA) was higher than anticipated (22). For oligometastatic prostate cancer, the multicenter, randomized Phase II study: Surveillance or Metastasis-Directed Therapy for Oligometastatic Prostate Cancer Recurrence (STOMP) showed that metastasis-directed therapy improved androgen-deprivation therapy free survival (23). Two separate randomized Phased II studies enrolling patients with oligometastatic non-small cell lung cancer (NSCLC) one from MD Anderson Cancer Center (MDACC), University of Colorado, London Health Sciences Centre (ON) $(24,25)$ and another from University of Texas Southwestern (26) -were closed early due to meeting the primary endpoint of progression-free survival benefit of SABR versus standard of care therapy alone. In both studies, patients with newly diagnosed primary NSCLC $(>90 \%$ of patients in both studies) and synchronously diagnosed oligometastases, who were randomized to the treatment arm, also underwent SABR or hypofractionated (15 fractions) radiotherapy to the primary tumor and regional nodal sites. Notable findings from the multi-institutional study (led by MDACC) included a significantly improved median OS (41 vs. 17 months, $\mathrm{P}=0.017$ ) and longer duration of time to develop new metastases (11.9 vs. 5.7 months, $\mathrm{P}=0.050)$ in the experimental/treatment arm. These trials have served as the basis for the recently opened NRG LU002 Phase IIR/III randomized controlled trial (NCT03137771) with a planned accrual of 300 patients. The UK "Stereotactic 
Ablative Radiotherapy for Oligometastatic Non-small Cell Lung Cancer (SARON)", with a planned accrual of 340 patients (NCT02417662), is another ongoing randomized study specific for NSCLC.

Many studies (in addition to the aforementioned ones) of metastasis-directed therapy for oligometastases, have been, or will be accruing patients; including other randomized studies $(27,28)$. The NRG BR002 Phase IIR/ III study is randomizing women with $\leq 4$ oligometastases from breast cancer to standard of care systemic therapy with or without upfront SABR or resection, with a target accrual of 360 patients, and has recently met the phase II accrual goal (personal communication from BR002 principal investigator S. Chmura) (NCT02364557). A study from the Institut Gustave Roussy- "Superiority of Stereotactic Body Radiation Therapy in Patients With Breast Cancer (STEREO-SEIN)"-is also randomizing women (target accrual 280 patients with $\leq 5$ oligometastases) with oligometastatic breast cancer (NCT02089100). The UK and Australian "Conventional Care Versus Radioablation (Stereotactic Body Radiotherapy) for Extracranial Oligometastases (CORE)" study (sponsored by the Royal Marsden Hospital; NCT02759783) plans to randomize 245 patients with 3 or fewer oligometastases from breast, prostate or NSCLC primary cancer, to receive or not receive SABR. The Randomized Phase II Study of Stereotactic Ablative Body Radiotherapy for Metastases to the Lung (TROG 13.01 SAFRON II) includes patients with any primary site, with the randomization being between 1 or 4 fractions of SABR.

One area of investigation not studied in in SABRCOMET is the role of incorporating immunotherapy in the treatment of oligometastases, a treatment paradigm with great potential. Immunotherapy in and of itself can afford prolonged disease-free survival in patients with metastatic disease; the addition of ablative doses of radiotherapy may potentiate the immune response via altering tumor stroma (29) and augmenting the immunogenic response (1,30-33), via promotion of tumor antigen presentation, chemokine/cytokine-mediated $\mathrm{T}$ cell infiltration, upregulation of adhesion molecules and other mechanisms. Therefore, patients with oligometastases may be particularly well-suited for combined SABR and immunotherapy $(1,31,33)$. Some speculate that the synergistic effect of SABR and immunotherapy may occur across many types of cancer. Notably, the NRG LU002 study has added immunotherapy as a stratification factor, and NRG BR002 hopes to incorporate immunotherapy-related investigations into a third arm if the trial proceeds to the phase 3 portion. The accruing SABR-COMET 3 and 10 studies will stratify patients by immunotherapy/targeted therapy $v s$. cytotoxic chemotherapy $v s$. observation (11). Several other studies of combining SABR with immunotherapy are accruing, particularly for patients with oligometastatic NSCLC (27), including studies from the University of Wisconsin (NCT03275597), European Thoracic Oncology Platform (CHESS; NCT03965468) and Shandong Cancer Hospital and Institute (NCT03557411). In a Phase II study from the University of Pennsylvania, 64 patients with 1-4 oligometastases from NSCLC underwent locally ablative therapy of metastases followed by pembrolizumab (34). The authors reported a median progression-free survival of 19.1 months $v s$. a historical value of 6.6 months $(\mathrm{P}=0.005)$ and discussed a hypothesis generating analyses showing improved outcomes with positive $v s$. negative programmed death ligand 1 (PD-L1) expression [hazard ration $=3.10$ (95\% confidence interval, 0.88-10.93)]. Studies of SABR (to select sites) plus immunotherapy for patients with widespread metastases is another area of great promise (35), albeit outside the scope of discussion of the treatment of oligometastatic disease.

Additional areas of research have been focused on identifying molecular correlates to help identify patients who are most apt to benefit from metastasis-directed therapy for oligometastases $(1,21,31)$. The NRG BR002 study plans to analyze circulating tumor cells and to store material for retrospective analyses of circulating tumor DNA and microRNA; NRG LU002 will also evaluate circulating tumor DNA. While such molecular correlates were not incorporated into the SABR-COMET study, future SABR-COMET studies plan to analyze circulating tumor cells, cell-free DNA, tumor DNA and immunological predictors of response (11).

In conclusion, the SABR-COMET study represents a major advance in understanding the potential benefit of metastasis-directed radiotherapy for patients with oligometastases. Clearly, future studies are needed, and ongoing-specifically disease-specific randomized Phase III studies, and studies incorporating molecular and biologic correlates to outcomes.

\section{Acknowledgments}

None. 


\section{Footnote}

Conflicts of Interest: The authors have no conflicts of interest to declare.

Ethical Statement: The authors are accountable for all aspects of the work in ensuring that questions related to the accuracy or integrity of any part of the work are appropriately investigated and resolved.

\section{References}

1. Weichselbaum RR. The 46th David A. Karnofsky Memorial Award Lecture: OligometastasisFrom Conception to Treatment. J Clin Oncol 2018:JCO1800847.

2. Salama JK, Milano MT. Radical irradiation of extracranial oligometastases. J Clin Oncol 2014;32:2902-12.

3. Ahmed KA, Torres-Roca JF. Stereotactic Body Radiotherapy in the Management of Oligometastatic Disease. Cancer Control 2016;23:21-9.

4. Rubin P, Green J. Solitary Metastases. Springfield, IL: C. C. Thomas 1968.

5. Rosenberg SA. Surgical treatment of metastatic cancer. Philadelphia, PA: Lippincott Williams \& Wilkins, 1987.

6. Peters LJ, Milas L, Fletcher GH. The role of radiation therapy in the curative treatment of metastatic disease. Symp Fundam Cancer Res 1983;36:411-20.

7. Hellman S, Weichselbaum RR. Oligometastases. J Clin Oncol 1995;13:8-10.

8. Palma DA, Olson RA, Harrow S, et al. Stereotactic ablative radiotherapy for the comprehensive treatment of oligometastatic tumors (SABR-COMET): Results of a randomized trial. Int J Radiat Oncol Biol Phys 2018;102:S3-4.

9. Rubinstein LV, Korn EL, Freidlin B, et al. Design issues of randomized phase II trials and a proposal for phase II screening trials. J Clin Oncol 2005;23:7199-206.

10. Wasserstein RL, Lazar NA. The ASA Statement on p-Values: Context, Process, and Purpose. The American Statistician 2016;70:129-33.

11. Palma DA, Olson R, Harrow S, et al. Stereotactic ablative radiotherapy for the comprehensive treatment of 4-10 oligometastatic tumors (SABR-COMET-10): study protocol for a randomized phase III trial. BMC Cancer 2019;19:816.

12. Milano MT, Katz AW, Schell MC, et al. Descriptive analysis of oligometastatic lesions treated with curative- intent stereotactic body radiotherapy. Int J Radiat Oncol Biol Phys 2008;72:1516-22.

13. Milano MT, Katz AW, Zhang H, et al. Oligometastases treated with stereotactic body radiotherapy: long-term follow-up of prospective study. Int J Radiat Oncol Biol Phys 2012;83:878-86.

14. Salama JK, Hasselle MD, Chmura SJ, et al. Stereotactic body radiotherapy for multisite extracranial oligometastases: final report of a dose escalation trial in patients with 1 to 5 sites of metastatic disease. Cancer 2012;118:2962-70.

15. Tong CC, Ko EC, Sung MW, et al. Phase II trial of concurrent sunitinib and image-guided radiotherapy for oligometastases. PLoS One 2012;7:e36979.

16. Sutera P, Clump DA, Kalash R, et al. Initial Results of a Multicenter Phase II Trial of Stereotactic Ablative Radiation Therapy for Oligometastatic Cancer. Int J Radiat Oncol Biol Phys 2019;103:116-22.

17. Milano MT, Katz AW, Zhang H, et al. Oligometastatic breast cancer treated with hypofractionated stereotactic radiotherapy: Some patients survive longer than a decade. Radiother Oncol 2019;131:45-51.

18. Aujla KS, Katz AW, Singh DP, et al. Hypofractionated Stereotactic Radiotherapy for Non-breast or Prostate Cancer Oligometastases: A Tail of Survival Beyond 10 Years. Front Oncol 2019;9:111.

19. Hong JC, Ayala-Peacock DN, Lee J, et al. Classification for long-term survival in oligometastatic patients treated with ablative radiotherapy: A multi-institutional pooled analysis. PLoS One 2018;13:e195149.

20. Rieber J, Streblow J, Uhlmann L, et al. Stereotactic body radiotherapy (SBRT) for medically inoperable lung metastases-A pooled analysis of the German working group "stereotactic radiotherapy". Lung Cancer 2016;97:51-8.

21. Wong AC, Watson SP, Pitroda SP, et al. Clinical and molecular markers of long-term survival after oligometastasis-directed stereotactic body radiotherapy (SBRT). Cancer 2016;122:2242-50.

22. Ruers T, Punt C, Van Coevorden F, et al. Radiofrequency ablation combined with systemic treatment versus systemic treatment alone in patients with non-resectable colorectal liver metastases: a randomized EORTC Intergroup phase II study (EORTC 40004). Ann Oncol 2012;23:2619-26.

23. Ost P, Reynders D, Decaestecker K, et al. Surveillance or Metastasis-Directed Therapy for Oligometastatic Prostate Cancer Recurrence: A Prospective, Randomized, Multicenter Phase II Trial. J Clin Oncol 2018;36:446-53.

24. Gomez DR, Blumenschein GR Jr, Lee JJ, et al. Local 
consolidative therapy versus maintenance therapy or observation for patients with oligometastatic non-smallcell lung cancer without progression after first-line systemic therapy: a multicentre, randomised, controlled, phase 2 study. Lancet Oncol 2016;17:1672-82.

25. Gomez DR, Tang C, Zhang J, et al. Local Consolidative Therapy Vs. Maintenance Therapy or Observation for Patients With Oligometastatic Non-Small-Cell Lung Cancer: Long-Term Results of a Multi-Institutional, Phase II, Randomized Study. J Clin Oncol 2019;37:1558-65.

26. Iyengar P, Wardak Z, Gerber DE, et al. Consolidative Radiotherapy for Limited Metastatic Non-Small-Cell Lung Cancer: A Phase 2 Randomized Clinical Trial. JAMA Oncol 2018;4:e173501.

27. Al-Shafa F, Arifin AJ, Rodrigues GB, et al. A Review of Ongoing Trials of Stereotactic Ablative Radiotherapy for Oligometastatic Cancers: Where Will the Evidence Lead? Front Oncol 2019;9:543.

28. Correa RJ, Salama JK, Milano MT, et al. Stereotactic Body Radiotherapy for Oligometastasis: Opportunities for Biology to Guide Clinical Management. Cancer J 2016;22:247-56.

29. Menon H, Ramapriyan R, Cushman TR, et al. Role of

Cite this article as: Milano MT, Chowdhry AK, Salama JK, Chmura SJ. Signals from SABR-COMET time to move on to phase III studies. Ann Transl Med 2019;7(Suppl 8):S316. doi: 10.21037/atm.2019.09.152
Radiation Therapy in Modulation of the Tumor Stroma and Microenvironment. Front Immunol 2019;10:193.

30. Cushman TR, Gomez D, Kumar R, et al. Combining radiation plus immunotherapy to improve systemic immune response. J Thorac Dis 2018;10:S468-79.

31. Pitroda SP, Chmura SJ, Weichselbaum RR. Integration of radiotherapy and immunotherapy for treatment of oligometastases. Lancet Oncol 2019;20:e434-42.

32. Weichselbaum RR, Liang H, Deng L, et al. Radiotherapy and immunotherapy: a beneficial liaison? Nat Rev Clin Oncol 2017;14:365-79.

33. Onderdonk BE, Chmura SJ. The Yin and Yang of Cytoreductive SBRT in Oligometastases and Beyond. Front Oncol 2019;9:706.

34. Bauml JM, Mick R, Ciunci C, et al. Pembrolizumab After Completion of Locally Ablative Therapy for Oligometastatic Non-Small Cell Lung Cancer: A Phase 2 Trial. JAMA Oncol 2019. [Epub ahead of print].

35. Luke JJ, Lemons JM, Karrison TG, et al. Safety and Clinical Activity of Pembrolizumab and Multisite Stereotactic Body Radiotherapy in Patients With Advanced Solid Tumors. J Clin Oncol 2018;36:1611-8. 